\title{
Approximate Basket Options Valuation for a Jump-Diffusion Model
}

\author{
Guoping Xu \\ Department of Mathematics \\ Imperial College \\ London SW7 2AZ, UK \\ guoping.xu@citi.com \\ Harry Zheng \\ (corresponding author) \\ Department of Mathematics \\ Imperial College \\ London SW7 2BZ, UK \\ h.zheng@imperial.ac.uk \\ Tel: +442075948539
}

\begin{abstract}
In this paper we discuss the approximate basket options valuation for a jump-diffusion model. The underlying asset prices follow some correlated diffusion processes with idiosyncratic and systematic jumps. We suggest a new approximate pricing formula which is the weighted sum of Roger and Shi's lower bound and the conditional second moment adjustments. We show the approximate value is always within the lower and upper bounds of the option and is very sharp in our numerical tests.
\end{abstract}

Keywords Basket option pricing, jump-diffusion model, analytic approximation, conditional moment matching

Mathematics Subject Classification (2000) 91B28, 60G99

JEL Classification G13

Insurance Mathematics Classification IM12, IM20 


\section{Introduction}

A basket option is an exotic option whose payoff depends on the value of a portfolio of assets. Basket options are in general difficult to price and hedge due to the lack of analytic characterization of the distribution of the sum of correlated random variables. Monte Carlo simulation is simple and accurate but is very time-consuming to price basket options. The other pricing methods are the numerical PDE, lower and upper bounds, and analytic approximations, see Lord (2006) for details.

Most work in the literature assume that underlying asset prices follow geometric Brownian motions. The basket value is then the sum of correlated lognormal variables. The main idea of the analytic approximation method is to find a simple random variable to approximate the basket value and then to use it to get a closed-form pricing formula. The approximate random variable is required to match some moments of the basket value. Levy (1992) uses a lognormal variable, Posner and Milevsky (1998) a shifted lognormal variable, and Milevsky and Posner (1998) a reciprocal gamma variable. The main drawback of these approximations is that the error can only be estimated by numerical analysis.

Curran (1994) introduces the idea of conditioning variable and conditional moment matching. The option price is decomposed into two parts: one can be calculated exactly and the other approximately by conditional moment matching method. The conditioning approach can also be used to find the bounds of the basket option. Rogers and Shi (1995) derive the lower and upper bounds, Nielsen and Sandmann (2003) improve the upper bound. Dhaene et al. (2002a, 2002b) introduce the concept of comonotonicity and discuss the comonotonic lower and upper bounds, Vyncke et al. (2004) propose a two moment matching approximation with a convex combination of the comonotonic lower and upper bounds for Asian options, Vanmaele et al. (2004) suggest a similar approximation for basket options. See Deelstra et al. (2004) and Lord (2006) for further extensions and applications.

All work mentioned above assume the diffusion asset price model. Efforts have been made to extend to more general asset price models. Albrecher and Predota $(2002,2004)$ discuss variance-gamma and NIG Lévy processes, Flamouris and Giamouridis (2007) Bernoulli jump diffusion model, and Hobson et al. (2005) and Chen et al. (2008) model free pricing.

In this paper we assume the underlying asset prices follow some jumpdiffusion processes. The innovative feature of the model is that, apart from correlated Brownian motions, there are two types of Poisson jumps: a sys- 
tematic jump that affects all asset prices and idiosyncratic jumps that only affect specific asset prices. Such a model can characterize well the marketwide phenomenon and individual events. In correlation modelling this is a type of Marchall-Olkin exponential copulas. Since the basket value is no longer the sum of lognormal variables it is not clear what conditioning random variables one should use to approximate the basket value. The main contribution of the paper is that we derive a new approximation to the basket call option price. The approximation is the weighted sum of the lower bound and the conditional second moment adjustments and is guaranteed to lie in between the lower bound and the upper bound. The numerical tests show that the approximation is very tight in comparison with the Monte Carlo results.

The paper is organized as follows. Section 2 formulates the jumpdiffusion asset price model and reviews some results on approximation and bounds in diffusion asset price models. Section 3 discusses conditioning random variables and derives a new approximation formula for basket options and shows it is bounded. Section 4 elaborates the numerical implementation and does some numerical tests. Appendix contains the details of a constant which is needed in approximation.

\section{Model Formulation}

Assume $\left(\Omega, P, \mathcal{F}, \mathcal{F}_{t}\right)$ is a filtered risk-neutral probability space and $\mathcal{F}_{t}$ is the augmented natural filtration generated by correlated Brownian motions $W_{1}, \ldots, W_{n}$ with correlation matrix $\left(\rho_{i j}\right)$ and independent Poisson processes $N_{0}, \ldots, N_{n}$ with intensities $\lambda_{0} \ldots, \lambda_{n}$. Assume Brownian motions and Poisson processes are independent to each other. Assume the portfolio is composed of $n$ assets and the asset prices $S_{1}, \ldots, S_{n}$ satisfy the stochastic differential equations

$$
\frac{d S_{i}(t)}{S_{i}(t)}=r_{i} d t+\sigma_{i} d W_{i}(t)+h_{i}^{0} d\left[N_{0}(t)-\lambda_{0} t\right]+h_{i}^{1} d\left[N_{i}(t)-\lambda_{i} t\right],
$$

for $i=1, \ldots, n$, where $r_{i}=r-\delta_{i}$ and $r$ is the risk-free interest rate and $\delta_{i}$ are the continuous dividend yields of assets $i, \sigma_{i}$ are volatilities of assets $i$, and $h_{i}^{0}, h_{i}^{1}$ are percentage jump sizes of assets $i$ at time of jumps of Poisson processes $N_{0}$ and $N_{i}$, respectively. All coefficients are assumed to be constant. Solutions to equations (1) are given by

$$
S_{i}(t)=S_{i}(0) e^{\left(r_{i}-\frac{1}{2} \sigma_{i}^{2}-h_{i}^{0} \lambda_{0}-h_{i}^{1} \lambda_{i}\right) t+\sigma_{i} W_{i}(t)+C_{i}^{0} N_{0}(t)+C_{i}^{1} N_{i}(t)},
$$


where $C_{i}^{0}=\ln \left(1+h_{i}^{0}\right)$ and $C_{i}^{1}=\ln \left(1+h_{i}^{1}\right)$.

Almost all the research in the literature on basket options pricing assumes that asset prices $S_{i}$ follow geometric Brownian motions (corresponding to $h_{i}^{0}=h_{i}^{1}=0$ for all $i$ ), which cannot explain asset prices jumps for unexpected sudden market events. The asset price dynamics (1) incorporates both systematic events and idiosyncratic events. More precisely, if an unexpected market event $N_{0}$ occurs at time $t$ then all underlying asset prices $S_{i}(t)$ have jumps of percentage sizes $h_{i}^{0}$ for $i=1, \ldots, n$, on the other hand, if an unexpected event $N_{i}$ occurs at time $t$, then only asset price $S_{i}(t)$ has a jump of percentage size $h_{i}^{1}$ but all other asset prices are not affected. In between jumps asset prices are driven by diffusion processes.

The basket value at time $t$ is given by

$$
A(t)=\sum_{i=1}^{n} w_{i} S_{i}(t)
$$

where $w_{i}$ are positive constant weights. The basket call option price at time 0 is given by

$$
\left.C_{0}=e^{-r T} \mathbf{E}[A(T)-K)^{+}\right]
$$

where $K$ is exercise price, $T$ maturity time, and $E$ risk-neutral expectation operator. In this paper the exercise time $T$ is fixed. To simplify the notation we will omit $T$ from now on, for example, we write $W_{i}$ instead of $W_{i}(T)$. The basket value at time $T$ can be written as

$$
A=\sum_{i=1}^{n} a_{i} e^{\sigma_{i} W_{i}+C_{i}^{0} N_{0}+C_{i}^{1} N_{i}}
$$

where $a_{i}=w_{i} S_{i}(0) e^{\left(r_{i}-\frac{1}{2} \sigma_{i}^{2}-h_{i}^{0} \lambda_{0}-h_{i}^{1} \lambda_{i}\right) T}, W_{i}$ are normal variables with mean 0 and variance $T$, and $N_{i}$ are Poisson variables with parameters $\lambda_{i} T, i=$ $1, \ldots, n$.

Almost all work in the literature on Asian or basket options pricing assume the underlying asset prices follow lognormal processes, which corresponds to $h_{i}^{0}=h_{i}^{1}=0$ for all $i$ in our model setup. Since the approach for Asian options can be easily adapted to basket options, and vice versa, we do not differentiate these two types of options, even though some techniques are originally developed for Asian options. We now review some well-known approaches in approximation and error bound estimation for the pure diffusion case.

Levy (1992) approximates the basket value $A$ with a lognormal variable which has the same first two moments as those of $A$ and derives the approximate closed-form pricing formula for $C_{0}$. Posner and Milevsky (1998) extend 
that approach to a shifted lognormal variable which matches the first four moments of $A$. The results are very good when maturity $T$ and volatilities $\sigma_{i}$ are relatively small. The performance deteriorates as $T$ or $\sigma_{i}$ increases. The drawback of these moment-matched lognormal approximations is that the approximation error can only be estimated by numerical analysis.

Curran (1994) introduces the idea of conditioning random variables. Assume $\Lambda$ is a random variable which has strong correlation with $A$ and satisfies that $A \geq K$ whenever $\Lambda \geq d_{\Lambda}$ for some constant $d_{\Lambda}$. The basket option price can be decomposed as

$$
\mathbf{E}\left[(A-K)^{+}\right]=\mathbf{E}\left[(A-K) 1_{\left[\Lambda \geq d_{\Lambda}\right]}\right]+\mathbf{E}\left[(A-K)^{+} 1_{\left[\Lambda<d_{\Lambda}\right]}\right] .
$$

Curran (1994) chooses $\Lambda$ a normal variable (geometric average) and finds the closed-form expression for the the first part and uses the lognormal variable and the conditional moment matching technique (at the point of strick price $K$ ) to find the approximate value of the second part. Deelstra et al. (2004) extend the conditional moment matching approach further by finding a lognormal variable $\tilde{A}$ such that

$$
\mathbf{E}[\tilde{A} \mid \Lambda=\lambda]=\mathbf{E}[A \mid \Lambda=\lambda] \quad \text { and } \quad \operatorname{Var}(\tilde{A} \mid \Lambda=\lambda)=\operatorname{Var}(A \mid \Lambda=\lambda)
$$

for all $\lambda<d_{\Lambda}$.

Rogers and Shi (1995) use the conditioning variable $\Lambda$ and Jensen's inequality to derive the lower bound of $\mathbf{E}\left[(A-K)^{+}\right]$as

$$
\mathbf{E}\left[(\mathbf{E}[A \mid \Lambda]-K)^{+}\right]
$$

Nielsen and Sandmann (2003), see also Rogers and Shi (1995), derive the upper bound of $\mathbf{E}\left[(A-K)^{+}\right]$as

$$
\mathbf{E}\left[(\mathbf{E}[A \mid \Lambda]-K)^{+}\right]+\frac{1}{2} \mathbf{E}\left[\operatorname{var}(A \mid \Lambda) 1_{\left[\Lambda<d_{\Lambda}\right]}\right]^{\frac{1}{2}} \mathbf{E}\left[1_{\left[\Lambda<d_{\Lambda}\right]}\right]^{\frac{1}{2}} .
$$

These bounds can be computed analytically.

Lord (2006) shows the conditional moment matching approximation of Deelstra et al. (2004) lies in between the lower and upper bounds and introduces the class of partially exact and bounded approximations.

The only work we are aware of on the jump-diffusion asset price model is the one by Flamouris and Giamouridis (2007). The basket contains two assets and each asset price may jump once. With this simplified setup the authors approximate basket value with a lognormal variable under each of the four cases (one may or may not jump and there is a combination of four 
cases) and approximate the basket option value by the weighted sum of the four approximating values.

For general jump-diffusion asset price models there are many open questions to be answered. For example, how should one choose the conditioning variable? Is the approximation guaranteed to lie in between the lower and upper bounds? How accurate and fast is the computation? etc. We will address these questions in the rest of the paper.

\section{Bounds and Approximation of Basket Options}

To use the conditioning variable approach to approximate the basket option price for a jump-diffusion asset price model, we need first to decide what conditioning variables to use. For a diffusion asset price model it is without exception in the literature to choose a normal variable as the conditioning variable, but it is not clear what one should choose for a jump-diffusion asset price model. From (2) we have

$$
\begin{aligned}
A & \geq \sum_{i=1}^{n} a_{i}\left(1+\sigma_{i} W_{i}+C_{i}^{0} N_{0}+C_{i}^{1} N_{i}\right) \\
& \geq c+m_{0} N_{0}+m_{2} N+\sigma W
\end{aligned}
$$

where $c=\sum_{i=1}^{n} a_{i}, m_{0}=\sum_{i=1}^{n} a_{i} C_{i}^{0}, m_{2}=\min _{1 \leq i \leq n}\left(a_{i} C_{i}^{1}\right)$, and $\sigma^{2}=$ $\sum_{i=1}^{n} \sum_{j=1}^{n} a_{i} a_{j} \rho_{i j} \sigma_{i} \sigma_{j} T$ are constant, and $N_{0}$ and $N=\sum_{i=1}^{n} N_{i}$ are Poisson variables with parameters $\lambda_{0} T$ and $\lambda T=\sum_{i=1}^{n} \lambda_{i} T$, respectively, and $W=$ $\frac{1}{\sigma} \sum_{i=1}^{n} a_{i} \sigma_{i} W_{i}$ is a standard normal variable. Note that $N_{0}, N$ and $W$ are independent to each other.

If we choose $X=\left(N_{0}, N, W\right)$ and define $\phi(X)=m_{0} N_{0}+m_{2} N+\sigma W$ and $d_{X}=K-c$ then we have $A \geq K$ whenever $\phi(X) \geq d_{X}$. Therefore $X$ can be a conditioning variable. The motivation for this choice is that we want to extract as much as possible the information of normal variables and Poisson variables. The reason we choose two Poisson variables $N_{0}$ and $N$ instead of combining them together is that $N_{0}$ is a common shock which has much greater impact on basket value $A$ than any individual shock $N_{i}$. This gives much better approximation with minimal increase of computation load.

The method of finding the lower and upper bounds of basket option price in Rogers and Shi (1995) and Nielsen and Sandmann (2003) works for the general jump-diffusion asset price model (1) by conditioning on $\{\phi(X) \geq$ $\left.d_{X}\right\}$. This leads to

$$
\mathrm{LB} \leq \mathbf{E}\left[(A-K)^{+}\right] \leq \mathrm{UB}
$$


where

$$
\begin{aligned}
\mathrm{LB} & =\mathbf{E}\left[(\mathbf{E}[A \mid X]-K)^{+}\right] \\
\mathrm{UB} & =L B+\frac{1}{2} \mathbf{E}\left[\operatorname{var}(A \mid X) 1_{\left[\phi(X)<d_{X}\right]}\right]^{\frac{1}{2}} \mathbf{E}\left[1_{\left[\phi(X)<d_{X}\right]}\right]^{\frac{1}{2}} .
\end{aligned}
$$

We have done some numerical tests for the lower and upper bounds of the jump-diffusion asset price process, see the next section. The results show that the lower bound is in general very tight whereas the upper bound is not sharp and can have large deviations to the exact value. Curran (1994) uses the lower bound to approximate the basket option price.

Denote by $A^{X}=\mathbf{E}[A \mid X]$ the conditional expectation of $A$ given $X$. The error between the lower bound and the exact basket option value is given by

$$
\begin{aligned}
& \mathbf{E}\left[(A-K)^{+}\right]-L B \\
= & \mathbf{E}\left[(A-K)^{+} 1_{\left[\phi(X)<d_{X}\right]}\right]-\mathbf{E}\left[\left(A^{X}-K\right)^{+} 1_{\left[\phi(X)<d_{X}\right]}\right] .
\end{aligned}
$$

This shows the error is caused by replacing $A 1_{\left[\phi(X)<d_{X}\right]}$ with $A^{X} 1_{\left[\phi(X)<d_{X}\right]}$. A simple calculation shows that

$$
\begin{aligned}
\mathbf{E}\left[A 1_{\left[\phi(X)<d_{X}\right]}\right] & =\mathbf{E}\left[A^{X} 1_{\left[\phi(X)<d_{X}\right]}\right] \\
\operatorname{Var}\left(A 1_{\left[\phi(X)<d_{X}\right]}\right) & =\operatorname{Var}\left(A^{X} 1_{\left[\phi(X)<d_{X}\right]}\right)+\mathbf{E}\left[\operatorname{Var}(A \mid X) 1_{\left[\phi(X)<d_{X}\right]}\right] .
\end{aligned}
$$

Therefore, the lower bound matches the first moment, but not the second moment. If we can find a random variable which matches the first two moments of $A 1_{\left[\phi(X)<d_{X}\right]}$ then we may reduce the error and improve the accuracy. We now look for such a random variable and find the properties it must hold. Let $\varepsilon$ be a random variable independent of $A$ and $X$ and satisfy the following two equations

$$
\begin{aligned}
\mathbf{E}\left[A 1_{\left[\phi(X)<d_{X}\right]}\right] & =\mathbf{E}\left[\left(A^{X}+\varepsilon\right) 1_{\left[\phi(X)<d_{X}\right]}\right] \\
\operatorname{Var}\left(A 1_{\left[\phi(X)<d_{X}\right]}\right) & =\operatorname{Var}\left(\left(A^{X}+\varepsilon\right) 1_{\left[\phi(X)<d_{X}\right]}\right)
\end{aligned}
$$

From (3), (5) and the independence of $\varepsilon$ and $X$ we get

$$
\mathbf{E}[\varepsilon]=0 .
$$

(7) and the independence of $\varepsilon$ and $X$ imply

$$
\begin{aligned}
& \operatorname{Var}\left(\left(A^{X}+\varepsilon\right) 1_{\left[\phi(X)<d_{X}\right]}\right) \\
= & \mathbf{E}\left[\left(A^{X}+\varepsilon\right)^{2} 1_{\left[\phi(X)<d_{X}\right]}\right]-\left(\mathbf{E}\left[\left(A^{X}+\varepsilon\right) 1_{\left[\phi(X)<d_{X}\right]}\right)^{2}\right. \\
= & \mathbf{E}\left[\left(A^{X}\right)^{2} 1_{\left[\phi(X)<d_{X}\right]}\right]+\mathbf{E}\left[\varepsilon^{2} 1_{\left[\phi(X)<d_{X}\right]}\right]-\left(\mathbf{E}\left[A^{X} 1_{\left[\phi(X)<d_{X}\right]}\right]\right)^{2} \\
= & \operatorname{Var}\left(A^{X} 1_{\left[\phi(X)<d_{X}\right]}\right)+\mathbf{E}\left[\varepsilon^{2}\right] \mathbf{E}\left[1_{\left[\phi(X)<d_{X}\right]}\right] .
\end{aligned}
$$


From (4), (6), and (8) we get

$$
\mathbf{E}\left[\varepsilon^{2}\right]=\frac{\mathbf{E}\left[\operatorname{Var}(A \mid X) 1_{\left[\phi(X)<d_{X}\right]}\right]}{\mathbf{E}\left[1_{\left[\phi(X)<d_{X}\right]}\right]} \equiv \varepsilon_{0}^{2} .
$$

We can now present the main result of the paper.

\section{Theorem 1 Let}

$$
A C_{0}=\mathbf{E}\left[(A-K)^{+} 1_{\left[\phi(X) \geq d_{X}\right]}\right]+\sum_{i=1}^{3} p_{i} \mathbf{E}\left[\left(A^{X}+\alpha_{i}-K\right)^{+} 1_{\left[\phi(X)<d_{X}\right]}\right]
$$

where $p_{1}=1 / 6, p_{2}=2 / 3, p_{3}=1 / 6$, and $\alpha_{1}=-\sqrt{3} \varepsilon_{0}, \alpha_{2}=0, \alpha_{3}=\sqrt{3} \varepsilon_{0}$. Then

$$
\mathrm{LB} \leq A C_{0} \leq \mathrm{UB}
$$

Proof. Let $\varepsilon$ be a discrete random variable taking values $\alpha_{i}$ with probabilities $p_{i}$ for $i=1,2,3$. Then $\mathbf{E}[\varepsilon]=0$ and $\mathbf{E}\left[\varepsilon^{2}\right]=\varepsilon_{0}^{2}$, i.e., $\varepsilon$ satisfies (7) and (9). We can now show that the new approximation is bounded by the lower and upper bounds. We first derive the upper bound.

$$
\begin{aligned}
& \mathbf{E}\left[\left(A^{X}+\varepsilon-K\right)^{+} 1_{\left[\phi(X)<d_{X}\right]}\right] \\
\leq & \mathbf{E}\left[\left(A^{X}-K\right)^{+} 1_{\left[\phi(X)<d_{X}\right]}+\varepsilon^{+} 1_{\left[\phi(X)<d_{X}\right]}\right] \\
= & \mathbf{E}\left[\left(A^{X}-K\right)^{+} 1_{\left[\phi(X)<d_{X}\right]}\right]+\frac{1}{2} \mathbf{E}[|\varepsilon|] \mathbf{E}\left[1_{\left[\phi(X)<d_{X}\right]}\right] \\
\leq & \mathbf{E}\left[\left(A^{X}-K\right)^{+} 1_{\left[\phi(X)<d_{X}\right]}\right]+\frac{1}{2} \mathbf{E}\left[\varepsilon^{2}\right]^{\frac{1}{2}} \mathbf{E}\left[1_{\left[\phi(X)<d_{X}\right]}\right] \\
= & \mathbf{E}\left[\left(A^{X}-K\right)^{+} 1_{\left[\phi(X)<d_{X}\right]}\right]+\frac{1}{2} \mathbf{E}\left[\operatorname{Var}(A \mid X) 1_{\left[\phi(X)<d_{X}\right]}\right]^{\frac{1}{2}} \mathbf{E}\left[1_{\left[\phi(X)<d_{X}\right]}\right]^{\frac{1}{2}} .
\end{aligned}
$$

Since $\varepsilon$ is symmetric around 0, i.e., $F(x)+F(-x)=1$ where $F$ is the distribution function of $\varepsilon$, we can also estimate the lower bound.

$$
\begin{aligned}
& \mathbf{E}\left[\left(A^{X}+\varepsilon-K\right)^{+} 1_{\left[\phi(X)<d_{X}\right]}\right] \\
= & \int_{-\infty}^{\infty} \mathbf{E}\left[\left(A^{X}+\eta-K\right)^{+} 1_{\left[\phi(X)<d_{X}\right]}\right] d F(\eta) \\
= & \int_{0}^{\infty} \mathbf{E}\left[\left[\left(A^{X}+\eta-K\right)^{+}+\left(A^{X}-\eta-K\right)^{+}\right] 1_{\left[\phi(X)<d_{X}\right]}\right] d F(\eta) \\
\geq & 2 \int_{0}^{\infty} \mathbf{E}\left[\left(A^{X}-K\right)^{+} 1_{\left[\phi(X)<d_{X}\right]}\right] d F(\eta) \\
= & \mathbf{E}\left[\left(A^{X}-K\right)^{+} 1_{\left[\phi(X)<d_{X}\right]}\right]
\end{aligned}
$$

Therefore,

$$
\mathrm{LB} \leq \mathbf{E}\left[\left(A^{X}+\varepsilon-K\right)^{+} 1_{\left[\phi(X)<d_{X}\right]}\right]+\mathbf{E}\left[(A-K)^{+} 1_{\left[\phi(X) \geq d_{X}\right]}\right] \leq \mathrm{UB} .
$$


We have proved the result.

We choose $e^{-r T} A C_{0}$ to approximate the basket option value at time 0 . It is clear from the proof that Theorem 1 holds for any random variable $\varepsilon$ as long as it is symmetric and satisfies (7) and (9). A normal distribution seems a natural choice, but then one has to deal with a numerical integration. We choose $\varepsilon$ a discrete random variable taking values $-\sqrt{3} \varepsilon_{0}, 0, \sqrt{3} \varepsilon_{0}$ with probabilities $1 / 6,2 / 3,1 / 6$, respectively, which matches the first five moments of a normal variable. We can expect the behaviour of $\varepsilon$ is similar to that of a normal variable with the added advantage that we do not need to compute the numerical integration. This choice of $\varepsilon$ also shows that the lower bound plays a dominant role in the approximation with a weight $2 / 3$, the other two parts with a weight $1 / 6$ each may be explained as the adjustment to the lower bound for the second moment.

Since the basket call option price tends to 0 as strike price $K \rightarrow \infty$, we would expect the approximate price $e^{-r T} A C_{0}$ tends to 0 too. This is indeed the case as shown in the next result.

Theorem 2 The value $A C_{0}$ defined in (10) tends to 0 as strike price $K$ tends to infinity.

Proof. The proof is similar to Theorem 4 in Lord (2006). We can write $A C_{0}$ as

$$
A C_{0}=\mathbf{E}\left[(A-K)^{+} 1_{\left[\phi(X) \geq d_{X}\right]}\right]+\mathbf{E}\left[\left(A^{X}+\varepsilon-K\right)^{+} 1_{\left[\phi(X)<d_{X}\right]}\right]
$$

where $\varepsilon$ is a discrete random variable defined in Theorem 1 . Since the call price tends to 0 as $K \rightarrow \infty$ it is obvious that the first term of (11) tends to 0 as $K \rightarrow \infty$. We now estimate the second term of (11). Let $\bar{A}=A^{X}+\varepsilon$, then

$$
\begin{aligned}
0 & \leq \mathbf{E}\left[(\bar{A}-K)^{+} 1_{\left[\phi(X)<d_{X}\right]}\right] \leq \mathbf{E}\left[(\bar{A}-K)^{+}\right] \\
& =\int_{K}^{\infty} P(\bar{A}>x) d x \leq \int_{K}^{\infty} P(|\bar{A}| \geq x) d x \\
& \leq \int_{K}^{\infty} \frac{\mathbf{E}\left[\bar{A}^{2}\right]}{x^{2}} d x=\frac{\mathbf{E}\left[\bar{A}^{2}\right]}{K}
\end{aligned}
$$

We only need to show that $\mathbf{E}\left[\bar{A}^{2}\right]$ is finite, which then implies $\frac{\mathbf{E}\left[\bar{A}^{2}\right]}{K}$ tends to 0 as $K \rightarrow \infty$. Since $A^{X}$ and $\varepsilon$ are independent and $\mathbf{E}[\varepsilon]=0$ we have

$$
\mathbf{E}\left[\bar{A}^{2}\right]=\mathbf{E}\left[\left(A^{X}\right)^{2}\right]+\mathbf{E}\left[\varepsilon^{2}\right] .
$$


Furthermore, $\phi(X) \geq d_{X}$ implies $A \geq K$, we have $P\left(\phi(X) \geq d_{X}\right) \leq P(A \geq$ $K)$, or equivalently, $P\left(\phi(X)<d_{X}\right) \geq P(A<K) \rightarrow 1$ as $K \rightarrow \infty$. Therefore, $P\left(\phi(X)<d_{X}\right) \geq 1 / 2$ for $K$ sufficiently large. This gives

$\mathbf{E}\left[\varepsilon^{2}\right]=\frac{\mathbf{E}\left[\operatorname{Var}(A \mid X) 1_{\left[\phi(X)<d_{X}\right]}\right]}{\mathbf{E}\left[1_{\left[\phi(X)<d_{X}\right]}\right]} \leq 2 \mathbf{E}[\operatorname{Var}(A \mid X)]=2 \mathbf{E}\left[A^{2}\right]-2 \mathbf{E}\left[\left(A^{X}\right)^{2}\right]$.

Obviously, we have $\mathbf{E}\left[\bar{A}^{2}\right] \leq 2 \mathbf{E}\left[A^{2}\right]<\infty$ for $K$ sufficiently large. We are done.

\section{Implementation and Numerical Tests}

We need first to find the conditional expectation of the basket value $A$ given the conditioning variable $X=\left(N_{0}, N, W\right)=\left(n_{0}, k, y\right)$.

$$
\begin{aligned}
& \mathbf{E}\left[A \mid X=\left(n_{0}, k, y\right)\right] \\
= & \sum_{i=1}^{n} a_{i} \mathbf{E}\left[e^{C_{i}^{0} N_{0}+C_{i}^{1} N_{i}+\sigma_{i} W_{i}} \mid X=\left(n_{0}, k, y\right)\right] \\
= & \sum_{i=1}^{n} a_{i} \mathbf{E}\left[e^{C_{i}^{0} N_{0}} \mid N_{0}=n_{0}\right] \mathbf{E}\left[e^{C_{i}^{1} N_{i}} \mid N=k\right] \mathbf{E}\left[e^{\sigma_{i} W_{i}} \mid W=y\right] .
\end{aligned}
$$

Here we have used the independence of $N_{0}, N, W$. For $N=\sum_{i=1}^{n} N_{i}$ define $\bar{N}_{i}=N-N_{i}$, then $\bar{N}_{i}$ is a Poisson variable with parameter $\bar{\lambda}_{i} T:=\left(\lambda-\lambda_{i}\right) T$ and is independent of $N_{i}$. From

$$
\begin{aligned}
P\left(N_{i}=k_{i} \mid N=k\right) & =\frac{P\left(N_{i}=k_{i}\right) P\left(\bar{N}_{i}=k-k_{i}\right)}{P(N=k)} \\
& =\frac{k !}{k_{i} !\left(k-k_{i}\right) !}\left(\frac{\lambda_{i}}{\lambda}\right)^{k_{i}}\left(\frac{\bar{\lambda}_{i}}{\lambda}\right)^{k-k_{i}}
\end{aligned}
$$

we get

$$
\begin{aligned}
E\left[e^{C_{i}^{1} N_{i}} \mid N=k\right] & =\sum_{k_{i}=0}^{k} e^{C_{i}^{1} k_{i}} \frac{k !}{k_{i} !\left(k-k_{i}\right) !}\left(\frac{\lambda_{i}}{\lambda}\right)^{k_{i}}\left(\frac{\bar{\lambda}_{i}}{\lambda}\right)^{k-k_{i}} \\
& =\left(e^{C_{i}^{1}} \frac{\lambda_{i}}{\lambda}+\frac{\bar{\lambda}_{i}}{\lambda}\right)^{k} .
\end{aligned}
$$

For $W=\frac{1}{\sigma} \sum_{i=1}^{n} a_{i} \sigma_{i} W_{i}$, Deelstra et al. [2004] show that

$$
\mathbf{E}\left[e^{\sigma_{i} W_{i}} \mid W=y\right]=e^{\frac{1}{2}\left(\sigma_{i}^{2} T-R_{i}^{2}\right)+R_{i} y}
$$


where $R_{i}=\frac{1}{\sigma} \sum_{j=1}^{n} a_{j} \rho_{i j} \sigma_{i} \sigma_{j} T$. Therefore

$$
\mathbf{E}\left[A \mid X=\left(n_{0}, k, y\right)\right]=\sum_{i=1}^{n} A_{i}\left(n_{0}, k, y\right)
$$

and

$$
A_{i}\left(n_{0}, k, y\right)=a_{i} e^{\frac{1}{2}\left(\sigma_{i}^{2} T-R_{i}^{2}\right)} e^{C_{i}^{0} n_{0}}\left(e^{C_{i}^{1}} \frac{\lambda_{i}}{\lambda}+\frac{\bar{\lambda}_{i}}{\lambda}\right)^{k} e^{R_{i} y}
$$

We can now easily find the exact part of the basket option value.

$$
\begin{aligned}
& \mathbf{E}\left[(A-K) 1_{\left[\phi(X) \geq d_{X}\right]}\right] \\
= & \mathbf{E}\left[\mathbf{E}[A \mid X] 1_{\left[\phi(X) \geq d_{X}\right]}\right]-K P\left(\phi(X) \geq d_{X}\right) \\
= & \sum_{n_{0}=0}^{\infty} \sum_{k=0}^{\infty} P\left(N_{0}=n_{0}\right) P(N=k) \\
& \cdot\left(\sum_{i=1}^{n} \tilde{S}_{i}\left(n_{0}, k\right) \Phi\left(R_{i}-z\left(n_{0}, k\right)\right)-K \Phi\left(-z\left(n_{0}, k\right)\right)\right)
\end{aligned}
$$

where $\tilde{S}_{i}\left(n_{0}, k\right)=a_{i} e^{\frac{1}{2} \sigma_{i}^{2} T} e^{C_{i}^{0} n_{0}}\left(e^{C_{i}^{1}} \frac{\lambda_{i}}{\lambda}+\frac{\bar{\lambda}_{i}}{\lambda}\right)^{k}$ and $z\left(n_{0}, k\right)=\frac{d_{X}-m_{0} n_{0}-m_{2} k}{\sigma}$.

It is slightly more involved in finding the approximating part of the basket option value. Denote by $\alpha$ a constant with value $-\sqrt{3} \varepsilon_{0}$ or 0 or $\sqrt{3} \varepsilon_{0}$. Then

$$
\begin{aligned}
& \mathbf{E}\left[(\mathbf{E}[A \mid X]+\alpha-K)^{+} 1_{\left[\phi(X)<d_{X}\right]}\right] \\
= & \sum_{n_{0}=0}^{\infty} \sum_{k=0}^{\infty} P\left(N_{0}=n_{0}\right) P(N=k) \int_{-\infty}^{z\left(n_{0}, k\right)}\left[\sum_{i=1}^{n} A_{i}\left(n_{0}, k, y\right)+\alpha-K\right]^{+} d \Phi(y) .
\end{aligned}
$$

For fixed $n_{0}$ and $k$ we need to compute the integral

$$
\int_{-\infty}^{z\left(n_{0}, k\right)}\left[\sum_{i=1}^{n} A_{i}\left(n_{0}, k, y\right)+\alpha-K\right]^{+} d \Phi(y) .
$$

To avoid the numerical integration we do the following: for fixed $n_{0}, k, \alpha$, define a strictly convex function

$$
f(y)=\sum_{i=1}^{n} A_{i}\left(n_{0}, k, y\right)+\alpha-K .
$$

We want to find $y^{*}=y\left(n_{0}, k, \alpha\right)$ such that $f\left(y^{*}\right)=0$. Four cases may occur.

1. $R_{i}=0$ for all $i$. Then $f$ is a constant. 
2. $R_{i} \geq 0$ for all $i$ and $R_{i}>0$ for at least one $i$. Then $f$ is strictly increasing and has at most one root.

3. $R_{i} \leq 0$ for all $i$ and $R_{i}<0$ for at least one $i$. Then $f$ is strictly decreasing and has at most one root.

4. $R_{i}>0$ for at least one $i$ and $R_{i}<0$ for at least another $i$. Then $f$ is U-shaped and has at most two roots.

We may use a numerical search algorithm such as the Newton method to find the root of $f$ and then compare it with $z\left(n_{0}, k\right)$ to get the closed-form value of the integration. Lord (2006) has a similar discussion concerning the number of roots of the function $f$.

To illustrate the point, we assume $R_{i}>0$ for all $i$. Then $f(y)$ is strictly increasing and $f(-\infty)=\alpha-K$ and $f(\infty)=\infty$. If $\alpha \geq K$ then $f$ has no root and $f(y)>0$ for all $y$. The integral of (12) equals

$$
\sum_{i=1}^{n} \tilde{S}_{i}\left(n_{0}, k\right) \Phi\left(z\left(n_{0}, k\right)-R_{i}\right)+(\alpha-K) \Phi\left(z\left(n_{0}, k\right)\right) .
$$

If $\alpha<K$ then $f$ has a unique root $y^{*}$, which implies $f(y)<0$ for $y<y^{*}$. Therefore, if $z\left(n_{0}, k\right) \leq y^{*}$ then the integral of (12) is 0 . If $z\left(n_{0}, k\right)>y^{*}$ then the integral of (12) equals

$$
\int_{y^{*}}^{z\left(n_{0}, k\right)}\left(\sum_{i=1}^{n} A_{i}\left(n_{0}, k, y\right)+\alpha-K\right) d \Phi(y)
$$

which can be computed explicitly. We can similarly discuss and solve all the other cases and we leave the details to the reader.

The only term remains to be computed is $\varepsilon_{0}$. To do so we need to find $\mathbf{E}\left[\operatorname{Var}(A \mid X) 1_{\left[\phi(X)<d_{X}\right]}\right]$, which is tedious but straightforward. The result is listed in the appendix.

Table 1 lists the results for a heterogeneous portfolio of two assets with different jump intensities $\left(\lambda_{0}=2, \lambda_{1}=1, \lambda_{2}=0.5\right)$ and same proportional jump sizes $\left(h_{0}=h_{1}=h_{2}=-0.2\right)$ and volatilities $\left(\sigma_{1}=\sigma_{2}\right)$, and with initial portfolio value $A(0)=100$ and correlation coefficient of Brownian motions $\rho_{12}=0.3$. We have done the numerical tests for the combination of the following data: maturity $T=1$ and 3 , volatility $\sigma_{i}=0.2,0.5$ and 0.8 , moneyness is $0.9,1$ and 1.1. (The moneyness is defined by $K / \mathbf{E}[A(T)]$, see Deelstra et al. (2004) and Lord (2006) for details.) The number of simulation is 1 million for $T=1$ and 3 million for $T=3$. Table 1 contains 9 columns. The first column reports the option maturity, the second one 
the volatility, the third one the moneyness, the fourth one the Monte Carlo value with standard deviation in parentheses, the fifth one the approximate value suggested in this paper, the sixth one the lower bound, the seventh one the upper bound, the eighth one the reciprocal gamma value (Milevsky and Posner (1998)), and the ninth one the lognormal value (Levy (1992)). The total computation time for each case (excluding simulation) takes only a few seconds. Monte Carlo takes much longer to compute but provides the benchmark values.

It is clear that the approximate values are very close to Monte Carlo values under different scenarios. The last row lists the RMSEs (root mean squared errors, defined by $\left.\operatorname{RMSE}=\left(\frac{1}{n} \sum_{i=1}^{n}\left(\text { Price }_{i}-\mathrm{MC}_{i}\right)^{2}\right)^{1 / 2}\right)$ for approximate, lower and upper bound, reciprocal gamma, and lognormal values. It is clear that the approximation method suggested by this paper has superior performance in comparison with the other methods. The approximate values are always between the lower and upper bounds. It is interesting to note that the lognormal approximation produces surprisingly good results although underlying asset prices follow jump-diffusion processes and not just diffusion processes as in Levy (1992), but its values can fall outside the region of the lower and upper bounds.

Table 2 lists the numerical results of the same data as in Table 1 except the correlation coefficient of Brownian motions is changed to $\rho_{12}=0.7$. All methods have better performance (especially lower and upper bounds) than ones recorded in Table 1, except the reciprocal gamma method which becomes worse. The approximation method still has the least RMSE.

Table 3 and 4 list the results for a homogeneous portfolio of four assets with jump intensities $\lambda_{0}=\lambda_{i}=1$, proportional jump sizes $h_{0}=h_{i}=-0.2$, and correlation coefficients of Brownian motions $\rho_{i j}=0.3$ (Table 3 ) and 0.7 (Table 4 ) for $i, j=1,2,3,4$. It is again clear that the approximation method produces the values very close to those of Monte Carlo under different scenarios and has the best performance over all other methods.

\section{Appendix: Computation of $\varepsilon_{0}$}

Since

$$
\varepsilon_{0}=\left(\frac{\mathbf{E}\left[\operatorname{Var}(A \mid X) 1_{\left[\phi(X)<d_{X}\right]}\right]}{\mathbf{E}\left[1_{\left[\phi(X)<d_{X}\right]}\right]}\right)^{\frac{1}{2}}
$$

and

$$
\mathbf{E}\left[\operatorname{Var}(A \mid X) 1_{\left[\phi(X)<d_{X}\right]}\right]=\mathbf{E}\left[\left(\mathbf{E}\left[A^{2} \mid X\right]-(\mathbf{E}[A \mid X])^{2}\right) 1_{\left[\phi(X)<d_{X}\right]}\right]
$$


we only need to find each of these terms. Here are the results.

$$
\mathbf{E}\left[1_{\left[\phi(X)<d_{X}\right]}\right]=\sum_{n_{0}=0}^{\infty} \sum_{k=0}^{\infty} P\left(N_{0}=n_{0}\right) P(N=k) \Phi\left(z\left(n_{0}, k\right)\right)
$$

and

$$
\begin{aligned}
& \mathbf{E}\left[(\mathbf{E}[A \mid X])^{2} 1_{\left[\phi(X)<d_{X}\right]}\right] \\
= & \sum_{n_{0}=0}^{\infty} \sum_{k=0}^{\infty} P\left(N_{0}=n_{0}\right) P(N=k) \\
& \cdot\left(\sum_{i=1}^{n} \sum_{j=1}^{n} \tilde{S}_{i}\left(n_{0}, k\right) \tilde{S}_{j}\left(n_{0}, k\right) e^{R_{i} R_{j}} \Phi\left(z\left(n_{0}, k\right)-R_{i}-R_{j}\right)\right)
\end{aligned}
$$

and

$$
\begin{aligned}
& \mathbf{E}\left[\mathbf{E}\left[A^{2} \mid X\right] 1_{\left[\phi(X)<d_{X}\right]}\right] \\
= & \sum_{n_{0}=0}^{\infty} \sum_{k=0}^{\infty} P\left(N_{0}=n_{0}\right) P(N=k) \\
& \cdot\left(\sum_{i=1}^{n} a_{i}^{2} e^{2 \sigma_{i}^{2} T} e^{2 C_{i}^{0} n_{0}}\left(e^{2 C_{i}^{1}} \frac{\lambda_{i}}{\lambda}+\frac{\bar{\lambda}_{i}}{\lambda}\right)^{k} \Phi\left(z\left(n_{0}, k\right)-2 R_{i}\right)\right) \\
& +\sum_{n_{0}=0}^{\infty} \sum_{n_{i}=0}^{\infty} \sum_{k=0}^{\infty} P\left(N_{0}=n_{0}\right) P\left(N_{i}=n_{i}\right) P\left(\bar{N}_{i}=k\right) \\
& \cdot\left(\sum_{i \neq j} a_{i} a_{j} e^{\frac{1}{2} \sigma_{i j}^{2} T} e^{\left(C_{i}^{0}+C_{j}^{0}\right) n_{0}} e^{C_{i}^{1} n_{i}}\left(e^{C_{j}^{1}} \frac{\lambda_{j}}{\bar{\lambda}_{i}}+1-\frac{\lambda_{j}}{\bar{\lambda}_{i}}\right)^{k} \Phi\left(z\left(n_{0}, n_{i}, k\right)-R_{i}-R_{j}\right)\right)
\end{aligned}
$$

where $\sigma_{i j}^{2}=\sigma_{i}^{2}+\sigma_{j}^{2}+2 \sigma_{i} \sigma_{j} \rho_{i j}, z\left(n_{0}, n_{i}, k\right)=\frac{d_{X}-m_{0} n_{0}-m_{2} n_{i}-m_{2} k}{\sigma}$, and $z\left(n_{0}, k\right)=\frac{d_{X}-m_{0} n_{0}-k m_{2}}{\sigma}$.

Acknowledgement. We wish to thank the anonymous referee for the useful comments and suggestions.

\section{References}

[1] Albrecher, H., Predota, M., 2002. Bounds and approximations for discrete Asian options in a variance-gamma model. Grazer Math. Ber. 345, $35-57$.

[2] Albrecher, H., Predota, M., 2004. On Asian option pricing for NIG lévy processes. Journal of Computational and Applied Mathematics $172,153-168$. 
[3] Chen, X., Deelstra, G., Dhaene, J., Vanmaele, M., 2008. Static superreplicating strategies for a class of exotic options. Insurance: Mathematics and Economics 42, 1067-1085.

[4] Curran, M., 1994. Valuing Asian and portfolio options by conditioning on the geometric mean price. Management Science 40, 1705-1711.

[5] Deelstra, G., Liinev, J., Vanmaele, M., 2004. Pricing of arithmetic basket options by conditioning. Insurance: Mathematics and Economics $34,55-77$.

[6] Dhaene, J., Denuit, M., Goovaerts, M.J., Kaas, R., Vyncke, D., 2002a. The concept of comonotonicity in actuarial science and finance: theory. Insurance: Mathematics and Economics 31, 3-33.

[7] Dhaene, J., Denuit, M., Goovaerts, M.J., Kaas, R., Vyncke, D., 2002b. The concept of comonotonicity in actuarial science and finance: applications. Insurance: Mathematics and Economics 31, 133-161.

[8] Flamouris, D., Giamouridis, D., 2007. Approximate basket option valuation for a simplified jump process. Journal of Future Market 27, 819837.

[9] Hobson, D., Laurence, P., Wang, T., 2005. Static-arbitrage upper bounds for the prices of basket options. Quantitative Finance 5, 329342 .

[10] Levy, E., 1992. Pricing European average rate currency options. Journal of International Money and Finance 11, 474-491.

[11] Lord, R., 2006. Partially exact and bounded approximations for arithmetic Asian options. Journal of Computational Finance 10, 1-52.

[12] Milevsky, M.A., Posner, S.E., 1998. Asian options, the sum of lognormals, and the reciprocal Gamma distribution. Journal of Financial and Quantitative Analysis 33, 409-422.

[13] Nielsen, J.A., Sandmann, K., 2003. Pricing bounds on Asian options. Journal of Financial and Quantitative Analysis 38, 449-473.

[14] Posner, S.E., Milevsky, M.A., 1998. Valuing exotic options by approximating the SPD with higher moments. Journal of Financial Engineering $7,109-125$. 
[15] Rogers, L.C.G., Shi, Z., 1995. The value of an Asian option. Journal of Applied Probability 32, 1077-1088.

[16] Vanmaele M., Deelstra, G., Liinev, J., 2004. Approximation of stoploss premiums involving sums of lognormals by conditioning on two variables. Insurance: Mathematics and Economics 35, 343-367.

[17] Vyncke, D., Goovaerts, M.J., Dhaene, J., 2004. An accurate analytical approximation for the price of a European-style arithmetic Asian option. Finance 25, 121-139. 


\begin{tabular}{|c|c|c|c|c|c|c|c|c|}
\hline Time & Vol & M's & MC (stdev) & $\mathrm{AP}$ & LB & UB & $\mathrm{RG}$ & LN \\
\hline \multirow[t]{9}{*}{1} & \multirow[t]{3}{*}{0.2} & 0.9 & $19.49(0.01)$ & 19.48 & 19.46 & 20.09 & 18.10 & 18.73 \\
\hline & & 1 & $14.34(0.01)$ & 14.34 & 14.32 & 15.03 & 13.36 & 13.83 \\
\hline & & 1.1 & $10.28(0.01)$ & 10.28 & 10.26 & 11.04 & 9.79 & 10.06 \\
\hline & \multirow[t]{3}{*}{0.5} & 0.9 & $25.01(0.02)$ & 24.97 & 24.84 & 26.66 & 23.00 & 24.61 \\
\hline & & 1 & $20.55(0.02)$ & 20.49 & 20.36 & 22.39 & 18.81 & 20.03 \\
\hline & & 1.1 & $16.84(0.02)$ & 16.76 & 16.64 & 18.87 & 15.43 & 16.60 \\
\hline & \multirow[t]{3}{*}{0.8} & 0.9 & $32.62(0.02)$ & 32.42 & 32.05 & 35.82 & 28.57 & 32.49 \\
\hline & & 1 & $28.77(0.03)$ & 28.50 & 28.15 & 32.34 & 24.88 & 28.65 \\
\hline & & 1.1 & $25.42(0.03)$ & 25.10 & 24.77 & 29.40 & 21.79 & 25.33 \\
\hline \multirow[t]{9}{*}{3} & \multirow[t]{3}{*}{0.2} & 0.9 & $28.95(0.04)$ & 28.98 & 28.80 & 32.32 & 25.51 & 27.94 \\
\hline & & 1 & $24.71(0.04)$ & 24.72 & 24.55 & 28.10 & 21.56 & 23.81 \\
\hline & & 1.1 & $21.06(0.04)$ & 21.05 & 20.90 & 24.48 & 18.30 & 20.29 \\
\hline & \multirow[t]{3}{*}{0.5} & 0.9 & $38.80(0.03)$ & 39.03 & 37.95 & 47.46 & 31.90 & 38.38 \\
\hline & & 1 & $35.32(0.02)$ & 35.37 & 34.41 & 44.10 & 28.49 & 34.92 \\
\hline & & 1.1 & $32.23(0.02)$ & 32.13 & 31.28 & 41.14 & 25.58 & 31.85 \\
\hline & \multirow[t]{3}{*}{0.8} & 0.9 & $51.53(0.07)$ & 51.91 & 49.33 & 65.65 & 36.99 & 52.07 \\
\hline & & 1 & $48.87(0.05)$ & 48.81 & 46.51 & 63.48 & 33.96 & 49.42 \\
\hline & & 1.1 & $46.44(0.07)$ & 46.05 & 43.96 & 61.58 & 31.34 & 47.00 \\
\hline RMSE & & & & 0.18 & 1.06 & 7.30 & 7.02 & 0.53 \\
\hline
\end{tabular}

Table 1: Basket option values and bounds with varying maturity $T$, volatility $\sigma$, and moneyness. Data: number of assets $n=2$, correlation of Brownian motions $\rho_{12}=0.3$, jump intensities $\lambda_{0}=2, \lambda_{1}=1, \lambda_{2}=0.5$, jump sizes $h_{0}=h_{1}=h_{2}=-0.2$, and interest rate $r=0.05$. 


\begin{tabular}{|c|c|c|c|c|c|c|c|c|}
\hline Time & Vol & M's & MC (stdev) & $\mathrm{AP}$ & LB & UB & $\mathrm{RG}$ & $\mathrm{LN}$ \\
\hline \multirow[t]{9}{*}{1} & \multirow[t]{3}{*}{0.2} & 0.9 & $19.83(0.01)$ & 19.83 & 19.82 & 20.29 & 18.45 & 19.13 \\
\hline & & 1 & $14.76(0.01)$ & 14.76 & 14.76 & 15.23 & 13.75 & 14.26 \\
\hline & & 1.1 & $10.73(0.01)$ & 10.72 & 10.71 & 11.29 & 10.19 & 10.50 \\
\hline & \multirow[t]{3}{*}{0.5} & 0.9 & $26.53(0.01)$ & 26.51 & 26.48 & 27.40 & 24.20 & 26.16 \\
\hline & & 1 & $22.21(0.01)$ & 22.18 & 22.15 & 23.17 & 20.12 & 21.89 \\
\hline & & 1.1 & $18.56(0.01)$ & 18.54 & 18.51 & 19.63 & 16.80 & 18.31 \\
\hline & \multirow[t]{3}{*}{0.8} & 0.9 & $35.10(0.02)$ & 35.04 & 34.97 & 36.57 & 30.02 & 34.89 \\
\hline & & 1 & $31.40(0.02)$ & 31.33 & 31.27 & 33.04 & 26.45 & 31.20 \\
\hline & & 1.1 & $28.17(0.02)$ & 28.08 & 28.03 & 29.98 & 23.43 & 27.98 \\
\hline \multirow[t]{9}{*}{3} & \multirow[t]{3}{*}{0.2} & 0.9 & $29.55(0.01)$ & 29.56 & 29.47 & 31.98 & 25.97 & 28.58 \\
\hline & & 1 & $25.36(0.02)$ & 25.37 & 25.28 & 27.82 & 22.06 & 24.49 \\
\hline & & 1.1 & $21.76(0.01)$ & 21.75 & 21.68 & 24.23 & 18.82 & 21.01 \\
\hline & \multirow[t]{3}{*}{0.5} & 0.9 & $40.98(0.02)$ & 40.97 & 40.73 & 45.36 & 32.91 & 40.46 \\
\hline & & 1 & $37.64(0.02)$ & 37.58 & 37.37 & 42.09 & 29.58 & 37.13 \\
\hline & & 1.1 & $34.65(0.02)$ & 34.56 & 34.38 & 39.19 & 26.73 & 34.16 \\
\hline & \multirow[t]{3}{*}{0.8} & 0.9 & $54.48(0.04)$ & 54.21 & 53.92 & 59.97 & 37.49 & 54.27 \\
\hline & & 1 & $51.94(0.04)$ & 51.62 & 51.37 & 57.67 & 34.50 & 51.74 \\
\hline & & 1.1 & $49.65(0.05)$ & 49.27 & 49.03 & 55.60 & 31.91 & 49.43 \\
\hline RMSE & & & & 0.14 & 0.27 & 3.23 & 8.29 & 0.49 \\
\hline
\end{tabular}

Table 2: Basket option values and bounds with varying maturity $T$, volatility $\sigma$, and moneyness. Data: number of assets $n=2$, correlation of Brownian motions $\rho_{12}=0.7$, jump intensities $\lambda_{0}=2, \lambda_{1}=1, \lambda_{2}=0.5$, jump sizes $h_{0}=h_{1}=h_{2}=-0.2$, and interest rate $r=0.05$. 


\begin{tabular}{|c|c|c|c|c|c|c|c|c|}
\hline Time & Vol & M's & MC (stdev) & $\mathrm{AP}$ & LB & UB & RG & $\mathrm{LN}$ \\
\hline \multirow[t]{9}{*}{1} & \multirow[t]{3}{*}{0.2} & 0.9 & $16.32(0.01)$ & 16.32 & 16.29 & 16.83 & 15.40 & 15.74 \\
\hline & & 1 & $10.78(0.01)$ & 10.77 & 10.74 & 11.40 & 10.27 & 10.48 \\
\hline & & 1.1 & $6.66(0.01)$ & 6.66 & 6.63 & 7.39 & 6.67 & 6.71 \\
\hline & \multirow[t]{3}{*}{0.5} & 0.9 & $21.41(0.01)$ & 21.37 & 21.23 & 22.83 & 20.19 & 21.15 \\
\hline & & 1 & $16.67(0.01)$ & 16.61 & 16.46 & 18.33 & 15.70 & 16.48 \\
\hline & & 1.1 & $12.86(0.01)$ & 12.77 & 12.64 & 14.77 & 12.20 & 12.75 \\
\hline & \multirow[t]{3}{*}{0.8} & 0.9 & $28.33(0.02)$ & 28.16 & 27.72 & 31.43 & 25.74 & 28.27 \\
\hline & & 1 & $24.20(0.02)$ & 23.96 & 23.53 & 27.77 & 21.81 & 24.15 \\
\hline & & 1.1 & $20.68(0.02)$ & 20.40 & 19.98 & 24.77 & 18.56 & 20.65 \\
\hline \multirow[t]{9}{*}{3} & \multirow[t]{3}{*}{0.2} & 0.9 & $23.42(0.01)$ & 23.44 & 23.26 & 26.12 & 21.47 & 22.70 \\
\hline & & 1 & $18.74(0.01)$ & 18.74 & 18.57 & 21.50 & 17.13 & 18.17 \\
\hline & & 1.1 & $14.88(0.01)$ & 14.85 & 14.70 & 17.69 & 13.68 & 14.48 \\
\hline & \multirow[t]{3}{*}{0.5} & 0.9 & $32.94(0.02)$ & 32.98 & 32.07 & 39.38 & 28.72 & $\overline{32.72}$ \\
\hline & & 1 & $29.10(0.02)$ & 28.99 & 28.16 & 35.77 & 25.04 & 28.90 \\
\hline & & 1.1 & $25.75(0.02)$ & 25.52 & 24.76 & 32.67 & 21.96 & 25.59 \\
\hline & \multirow[t]{3}{*}{0.8} & 0.9 & $45.31(0.04)$ & 45.75 & 42.81 & 58.32 & 35.22 & 46.15 \\
\hline & & 1 & $42.29(0.04)$ & 42.40 & 39.60 & 56.22 & 32.06 & 43.15 \\
\hline & & 1.1 & $39.59(0.04)$ & 39.39 & 36.72 & 54.47 & 29.34 & 40.45 \\
\hline RMSE & & & & 0.17 & 1.20 & 6.63 & 4.65 & 0.46 \\
\hline
\end{tabular}

Table 3: Basket option values and bounds with varying maturity $T$, volatility $\sigma$, and moneyness. Data: number of assets $n=4$, correlation of Brownian motions $\rho_{i j}=0.3$, jump intensities $\lambda_{0}=\lambda_{i}=1$, jump sizes $h_{0}=h_{i}=-0.2$ for $i, j=1,2,3,4$, and interest rate $r=0.05$. 


\begin{tabular}{|c|c|c|c|c|c|c|c|c|}
\hline Time & Vol & M's & MC (stdev) & $\mathrm{AP}$ & LB & UB & $\mathrm{RG}$ & LN \\
\hline \multirow[t]{9}{*}{1} & \multirow[t]{3}{*}{0.2} & 0.9 & $16.99(0.01)$ & 16.99 & 16.97 & 17.39 & 16.09 & 16.49 \\
\hline & & 1 & $11.60(0.01)$ & 11.60 & 11.58 & 12.08 & 11.07 & 11.33 \\
\hline & & 1.1 & $7.55(0.01)$ & 7.55 & 7.53 & 8.11 & 7.47 & 7.55 \\
\hline & \multirow[t]{3}{*}{0.5} & 0.9 & $24.19(0.01)$ & 24.17 & 24.14 & 24.92 & 22.51 & 23.98 \\
\hline & & 1 & $19.71(0.01)$ & 19.69 & 19.66 & 20.56 & 18.26 & 19.55 \\
\hline & & 1.1 & $16.01(0.01)$ & 15.99 & 15.96 & 16.98 & 14.86 & 15.90 \\
\hline & \multirow[t]{3}{*}{0.8} & 0.9 & $32.89(0.01)$ & 32.82 & 32.77 & 34.19 & 28.75 & 32.77 \\
\hline & & 1 & $29.06(0.02)$ & 28.99 & 28.93 & 30.56 & 25.07 & 28.95 \\
\hline & & 1.1 & $25.74(0.02)$ & 25.66 & 25.61 & 27.43 & 21.99 & 25.64 \\
\hline \multirow[t]{9}{*}{3} & \multirow[t]{3}{*}{0.2} & 0.9 & $24.64(0.01)$ & 24.64 & 24.55 & 26.65 & 22.51 & 23.99 \\
\hline & & 1 & $20.09(0.01)$ & 20.08 & 20.00 & 22.16 & 18.28 & 19.56 \\
\hline & & 1.1 & $16.31(0.01)$ & 16.28 & 16.20 & 18.42 & 14.87 & 15.91 \\
\hline & \multirow[t]{3}{*}{0.5} & 0.9 & $37.20(0.02)$ & 37.13 & 36.98 & 40.14 & 31.14 & 36.91 \\
\hline & & 1 & $33.64(0.02)$ & 33.54 & 33.40 & 36.71 & 27.66 & 33.35 \\
\hline & & 1.1 & $30.49(0.02)$ & 30.37 & 30.24 & 33.69 & 24.71 & 30.22 \\
\hline & \multirow[t]{3}{*}{0.8} & 0.9 & $51.20(0.03)$ & 50.90 & 50.70 & 55.35 & 36.74 & 51.12 \\
\hline & & 1 & $48.48(0.03)$ & 48.15 & 47.96 & 52.97 & 33.70 & 48.41 \\
\hline & & 1.1 & $46.01(0.05)$ & 45.66 & 45.47 & 50.86 & 31.06 & 45.95 \\
\hline RMSE & & & & 0.14 & 0.24 & 2.49 & 6.75 & 0.29 \\
\hline
\end{tabular}

Table 4: Basket option values and bounds with varying maturity $T$, volatility $\sigma$, and moneyness. Data: number of assets $n=4$, correlation of Brownian motions $\rho_{i j}=0.7$, jump intensities $\lambda_{0}=\lambda_{i}=1$, jump sizes $h_{0}=h_{i}=-0.2$ for $i, j=1,2,3,4$, and interest rate $r=0.05$. 